\title{
Protective effect of erythropoietin on myocardial infarction in rats by inhibition of caspase-12 expression
}

\author{
SHAOXIANG WENG, XIAOQING ZHU, YUE JIN, TING WANG and HE HUANG \\ Department of Cardiology, Sir Run Run Shaw Hospital, School of Medicine, \\ Zhejiang University, Hangzhou 310003, P.R. China
}

Received April 6, 2011; Accepted June 1, 2011

DOI: $10.3892 /$ etm.2011.280

\begin{abstract}
In the present study, the myocardial protective effects of erythropoietin (EPO) by inhibition of the expression of caspase-12 were investigated in a myocardial infarction rat model. Thirty male SD rats were divided into three groups: sham-operation group, myocardial infarction group and EPO treatment group. The myocardial infarction model was created by ligating the left anterior descending coronary artery. The EPO treatment group was established by injecting rh-EPO $(1,000 \mathrm{IU} / \mathrm{kg})$ intraperitoneally every day after the operation, and the other two groups were injected with sodium chloride. Four weeks after induction of myocardial infarction, the left ventricular diastolic pressure (LVDP) was tested by Langendorff apparatus and the pathological changes were analyzed by $H \& E$ staining. Caspase-12 expression in the left ventricular myocardium was also measured by immunohistochemistry. Four weeks after induction of myocardial infarction, the improvement in heart function in the EPO treatment group was more distinct compared to that of the myocardial infarction group; LVDP was higher in the EPO treatment group compared to the myocardial infarction group, but lower compared to the control group. H\&E staining showed that the myocardial cells in the normal control group were aligned in order with a clear structure and were stained equably, while the myocardial cells in the myocardial infarction model rats lined up in disorder with an augmented cell body appearing to have many granules and interstitial fibrosis. Myocardial fibrosis and disorder were improved in the EPO treatment group. The expression of caspase-12 in the myocardial infarction group was also increased compared to the EPO treatment group rats. The results suggest that EPO improves heart function in myocardial infarction rats by down-regulating the expression of caspase-12, which may protect the myocardium by abrogating endoplasmic reticulum stress-mediated cardiomyocyte apoptosis and improving heart function.
\end{abstract}

Correspondence to: Dr Shaoxiang Weng, Sir Run Run Shaw Hospital, School of Medicine, Zhejiang University, Hangzhou 310003, P.R. China

E-mail: wengshaoxiang@163.com

Key words: erythropoietin, myocardial infarction, caspase-12

\section{Introduction}

Myocardial infarction results in insufficient blood supply, decreasing the function of myocardial cells and ventricular remodeling, eventually leading to heart failure (1). Myocardial cell apoptosis is a critical feature of myocardial infarction (2). Erythropoietin (EPO) is a glycoprotein hormone produced by the kidney and fetal liver (3). In recent studies, EPO was shown to have protective effects against ischemic injury in the brain (4), spinal cord (5), kidney (6), and even in the myocardium (7). Clinical application of EPO to treat anemia associated with congestive heart failure was found to significantly improve cardiac function in these patients (8), and increasing evidence suggests that EPO increases the ability of myocardial cells to endure ischemic tolerance of hypoxia. Current findings suggest that the protective effects of EPO on myocardial infarction depend on the anti-apoptotic effect of certain cytokines, but its exact mechanism remains unknown (9).

The endoplasmic reticulum (ER) has received much attention recently for its role in signal transduction relevant to cell survival and death. Myocardial infarction conditions induce $\mathrm{Ca}^{2+}$ overload and/or accumulation of unfolding or misfolding proteins within the ER. ER stress induces multiple responses, including adaptive changes in translation, protein folding, secretion and degradation. Prolonged ER stress triggers apoptosis by induction of C/EBP homologous protein (CHOP), activation of c-JUN NH2-terminal kinase (JNK) and finally production of pro-apoptotic factors (caspase-12), which may contribute to the development of diabetic nephropathy (10). In addition, caspase-12 causes ER stress-induced apoptosis, which is a feature of ischemia/reperfusion (I/R) injury, and thus suppressing ER stress may protect the myocardium from $\mathrm{I} / \mathrm{R}$ injury (11).

In the present study, we hypothesized that the protective effect of EPO against ischemic injury in the myocardium was through the reduction of ER stress-induced apoptosis. We induced myocardial infarction in a rat model by ligating the left anterior descending coronary artery and examined systolic and diastolic function 4 weeks after myocardial infarction, as well as myocardial fibrosis, apoptosis, angiogenesis and expression of caspase-12 in myocardial infarction myocardium, non-infarcted myocardium and myocardial infarction myocardium treated with EPO. The aim of this study was to determine the relationship between a decrease in caspase-12 
expression in the heart and the protective effects of EPO on myocardial infarction.

\section{Materials and methods}

Materials. Sprague-Dawley male rats were purchased from the Experimental Animal Centre of Zhejiang University (Hangzhou, China). Recombinant human erythropoietin (rhEPO) was obtained from Shenyang Sunshine Pharmaceutical Co., Ltd. (Shenyang, China). Monoclonal caspase-12 antibody was purchased from Abcam Company (Cambridge, MA, USA). Langendorff heart perfusion apparatus was purchased from Nanjing Medease Science and Technology Co., Ltd. (Nanjing, China).

Animal model induction. Thirty male Sprague-Dawley rats with a body weight of $\sim 210 \mathrm{~g}$ were used for this study and were randomly divided into three groups (10 rats/group): sham-operation group, myocardial infarction group and EPO treatment group. Under pentobarbital anesthesia, rats were intubated and mechanically ventilated with a Harvard respirator. Physiological arterial blood gas levels were maintained by adjusting the respirator and oxygen supplement. Hearts were exposed via the left thoracotomy, and a coronary snare was prepared around the left anterior artery. In the myocardial infarction and EPO treatment groups, the left anterior descending coronary artery was ligatured between the pulmonary cone and the left atrial appendage, and the left anterior descending coronary artery blood supply region became white. Subsequently, the rats were intraperitoneally injected with $4.0 \times 10^{6} \mathrm{U}$ of penicillin to prevent infection, and revived at $30^{\circ} \mathrm{C}$, while the sham-operation rats only received thoracotomy. After the operation, the EPO group rats received an intraperitoneal injection of EPO $\left(1 \times 10^{3} \mathrm{IU} /\right.$ $\mathrm{kg} / \mathrm{day}$ ), while the sham-operation and myocardial infarction group rats received an intraperitoneal injection of normal saline $(1 \mathrm{ml} / \mathrm{kg} /$ day $)$ for 4 weeks.

Hemodynamic measurements. Coronary perfusion using the Langendorff system with modified Krebs-Henseleit $(\mathrm{KH})$ solution containing $118.5 \mathrm{NaCl}, 4.7 \mathrm{KCl}, 1.2 \mathrm{MgSO}_{4}, 1.8 \mathrm{CaCl}_{2}$, $24.8 \mathrm{NaHCO}_{3}, 1.2 \mathrm{KH}_{2} \mathrm{PO}_{4}$ and 10 glucose (in $\mathrm{mM}$ ) was carried out. After 4 weeks, five isolated hearts in each group were extracted. A KH buffer-filled latex balloon was inserted into the left ventricle (LV) and was adjusted to $5-10 \mathrm{mmHg}$ of the left ventricular end-diastolic pressure (LVEDP) at the beginning of the experiment. Each isolated heart was perfused with $37^{\circ} \mathrm{C}$ oxygenated $\left(95 \% \mathrm{O}_{2}-5 \% \mathrm{CO}_{2}\right)$ modified $\mathrm{KH}$ buffer. Left ventricular systolic pressure (LVSP) and LVEDP were recorded with the Langendorff heart perfusion apparatus system by averaging the values for ten beats. Left ventricular diastolic pressure (LVDP) was calculated as follows: LVDP = LVSP - LVEDP.

Immunohistochemistry and $H \& E$ staining. Immunohistochemistry was used to localize the caspase-12 antigen. Five rats from each group were anesthetized with a lethal dose of pentobarbital. Their thoracic cavities were then opened and perfused intracardially with normal saline. Following saline perfusion, the animals were perfused with $300-400 \mathrm{ml}$ of fixative containing $4 \%$ paraformaldehyde in $0.1 \mathrm{M}$ phosphate buffer ( $\mathrm{pH}$ 7.4). After perfusion, the heart of each rat was removed, fixed in the same

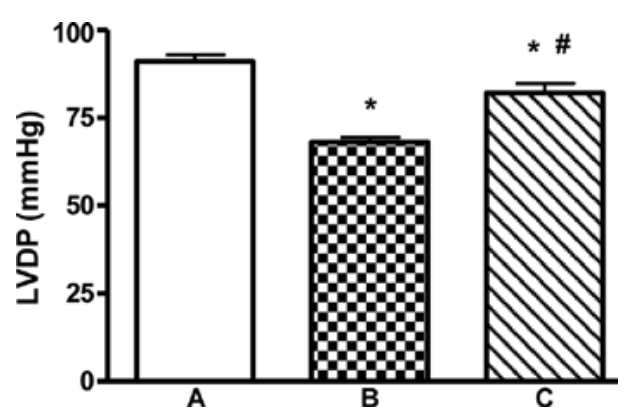

Figure 1. LVDP in each group 4 weeks after operation. (A) Control, (B) myocardial infarction and (C) EPO treatment rats. ${ }^{*} \mathrm{P}<0.01$ vs. control; ${ }^{\#} \mathrm{P}<0.01$ vs. myocardial infarction.

fixative for $4 \mathrm{~h}$ and then placed in $30 \%$ phosphate-buffered sucrose until the tissue sank. Tissue sections $(8 \mu \mathrm{m})$ were cut on a freezing microtome through the coronary planes of the heart. Sections were then rinsed in $0.01 \mathrm{M}$ phosphate-buffered saline (PBS) and mounted onto $0.02 \%$ poly-l-lysine-coated slides.

Tissue sections were washed in PBS, incubated in $1 \%$ bovine serum albumin (BSA) for $30 \mathrm{~min}$, and then incubated overnight at $4^{\circ} \mathrm{C}$ in the primary antibody (monoclonal caspase-12 antibody) plus $1 \%$ BSA in PBS. The dilution of the primary antibody was 1:100. Control sections were incubated in PBS alone. The next day, the sections were incubated in a biotinylated goat anti-mouse secondary antibody (diluted to 1:200 in PBS), and subsequently in an avidin-horseradish peroxidase solution. Immunolabelling was visualized with $0.05 \%$ DAB (Sigma, St. Louis, MO, USA) plus $0.3 \% \mathrm{H}_{2} \mathrm{O}_{2}$ in PBS. The sections were then dehydrated through ethanol and xylene before being cover-slipped with Permount ${ }^{\mathrm{TM}}$. H\&E staining was also applied to determine morphological changes.

Image analysis. For immunohistochemistry, the left ventricular tissues were selected on each slide and examined at a magnification of $\mathrm{x} 400$ with a UTHSCSA Image Tool 3.0 (University of Texas Medical School at San Antonio, San Antonio, TX, USA). The number and optical density of the caspase-12-positive cells were measured.

Statistical analysis. All data are the means \pm SD. Statistical analysis was performed using SPSS $^{\circledR}$ for Windows ${ }^{\circledR}$ version 12.0 statistical software (SPSS Inc., St. Louis, MO, USA). The significance of any differences between the groups was evaluated using the one-way ANOVA. P-values $<0.05$ were considered significant.

\section{Results}

Hemodynamic parameters. LVDP of the myocardial infarction group rats was significantly decreased to $68.5 \pm 4.1 \mathrm{mmHg}$, while that in the sham-operation rats was $91.3 \pm 7.9 \mathrm{mmHg}$. Treatment with EPO for 4 weeks caused a statistically significant increase in cardiac functional recovery improving the LVDP to $82.9 \pm 8.35$ $\mathrm{mmHg}(\mathrm{P}<0.01)$ in the EPO treatment group when compared to that in the myocardial infarction group (Fig. 1).

Heart pathological changes. As shown in Fig. 2B, infarction, myocardial fibrosis, the collagen deposition area and the 

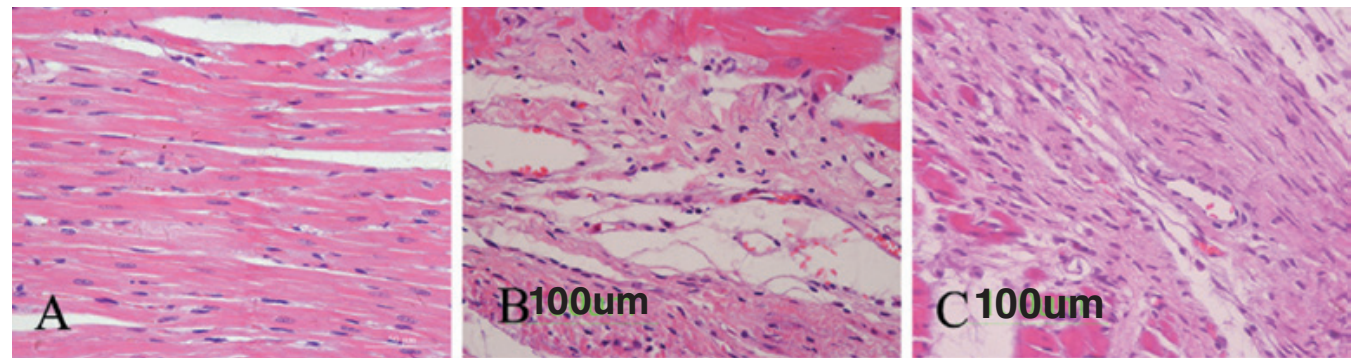

Figure 2. Pathological changes in the myocardium of (A) a control rat, (B) a myocardial infarction rat and (C) an EPO treatment rat 4 weeks after operation by H\&E staining.
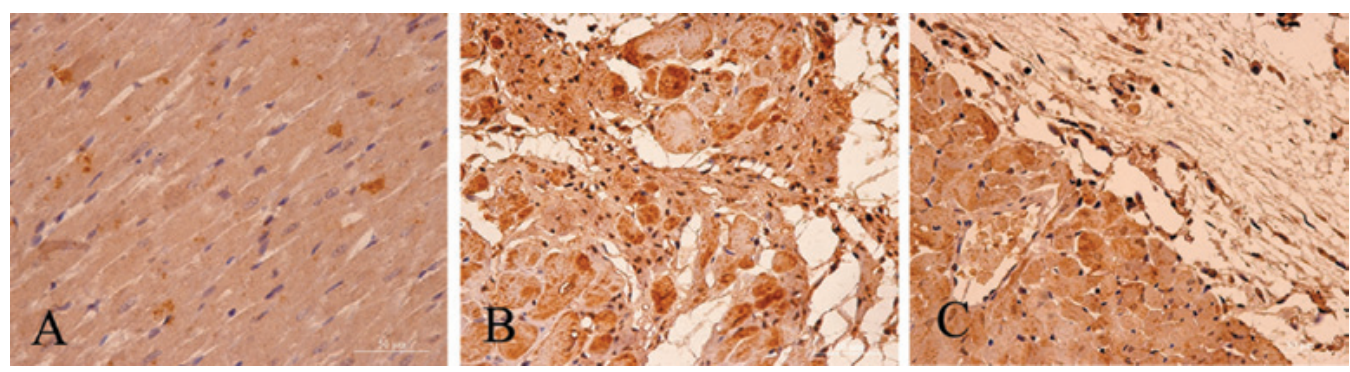

Figure 3. Light photomicrographs of caspase-12 immunohistochemical staining of the myocardium from (A) a control rat, (B) a myocardial infarction rat and (C) an EPO treatment rat.
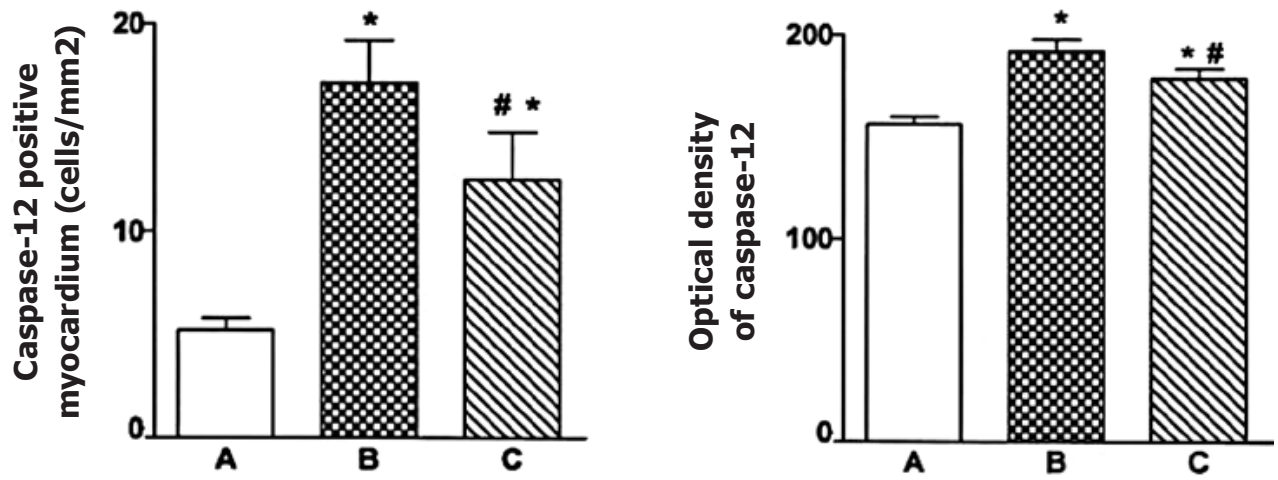

Figure 4. Comparison of the expression of caspase-12 in the myocardium of (A) a control rat, (B) a myocardial infarction rat and (C) an EPO treatment rat. ${ }^{*} \mathrm{P}<0.01$ vs. control group; ${ }^{*} \mathrm{P}<0.01$ vs. myocardial infarction group.

remnants of cardiac disorder were substantially severe in the myocardial infarction group rats. Fig. $2 \mathrm{C}$ demonstrates a notably higher number of small vessels around the infarcted myocardium in the EPO treatment group rats than in the myocardial infarction group rats. The myocardial interstitial fibrosis area was also improved in the EPO treatment group rats (Fig. 2).

Immunohistochemical staining of caspase-12 localization. Fig. 2 shows light photomicrographs of caspase-12 immunohistochemical staining of the myocardium in the three groups of rats. In heart tissues from the myocardial infarction rats, the myocardium underwent fibrosis and structural rarefaction. Myocardium that stained positive for caspase-12 showed buff-colored granules with DAB staining. The myocardium showed increased caspase-12 expression in the myocardial infarction group when compared to the control group. Also, the EPO-treated rats had a significantly decreased number of caspase-12-positive cells and decreased optical density of the caspase-12 immunostained myocardium compared to the myocardial infarction rats $(\mathrm{P}<0.01)$ (Figs. 3 and 4).

\section{Discussion}

The ER is the site for the proper folding and assembly of membrane-localized proteins and those destined for secretion. It is also a regulator of intracellular calcium (12). Various pathophysiological conditions induce $\mathrm{Ca}^{2+}$ overload and/or accumulation of unfolding or misfolding proteins within the ER, a condition referred to as ER stress (13). ER stress-induced apoptosis is believed to be associated with several pathological processes, including diabetes (14), ischemia-reperfusion injury (7), Alzheimer's disease and Parkinson's disease $(15,16)$. Researchers have already found that myocardial ischemia provokes ER stress which results in the apoptosis of myocar- 
dial and coronary endothelial cells; in addition, caspase-12 plays a major role in this apoptosis induction $(17,18)$. Our results demonstrated that the expression of caspase-12 was significantly elevated in the myocardial ischemic areas compared to the normal areas. Also, apoptosis of myocardial cells increased accompanied by myocardial fibrosis in the myocardial ischemic areas which caused cardiac dysfunction.

EPO has emerged as a promising candidate for the treatment of acute ischemic heart disease (7). Apart from its hematopoietic effects, EPO exhibits anti-apoptotic effects, particularly under ischemic conditions, and attenuates oxidative stress (19). The binding of EPO to the EPO receptor leads to a hemodimerization, with subsequent activation of janus kinase (26). EPO signaling involves multiple pathways, including activation of STAT 5, activation of proteins with Src homology, such as PI3 kinase, and activation of ras/MAP kinases $(20,21)$. In the present study, EPO improved myocardial contractility, reduced celluar damage and apoptosis, increased neovascularization, and improved and decreased the expression of caspase- 12 in a rat model of myocardial infarction. These results suggest that the mechanism of cardiac improvement by application of EPO may block signal transduction in ER stress via reduction of the expression of caspase-12, which has a beneficial effect on myocardial protection.

The present study used the Langendorff system to record the LVDP and immunohistochemistry to determine the expression of caspase-12 protein in the heart after 4 weeks of myocardial infarction induction, and to demonstrate the protective effect of EPO on myocardial infarction. There was less function reduction and structural damage to the heart in the EPO-treated group than in the myocardial infarction group. We demonstrated that EPO treatment of myocardial infarction prevents the appearance of subsequent clinical symptoms by reversing the functional and morphological abnormalities in the myocardial infarction heart in a rat model through abrogation of the myocardial infarction-induced increase in caspase-12 expression and prevented ER stress-induced apoptosis after myocardial infarction. This study provides the experimental basis for EPO treatment for myocardial infarction, while the exact mechanism of action will be the subject of further investigation.

\section{References}

1. McManus DD, Chinali M, Saczynski JS, et al: 30-Year trends in heart failure in patients hospitalized with acute myocardial infarction. Am J Cardiol 107: 353-359, 2011.

2. Takemura G, Ohno M, Hayakawa Y, et al: Role of apoptosis in the disappearance of infiltrated and proliferated interstitial cells after myocardial infarction. Circ Res 82: 1130-1138, 1998.
3. Weidemann A and Johnson RS: Nonrenal regulation of EPO synthesis. Kidney Int 75: 682-688, 2009.

4. Iwai M, Cao G, Yin W, et al: Erythropoietin promotes neuronal replacement through revascularization and neurogenesis after neonatal hypoxia/ischemia in rats. Stroke 38: 2795-2803, 2007.

5. Matis GK and Birbilis TA: Erythropoietin in spinal cord injury. Eur Spine J 18: 314-323, 2009.

6. Breggia AC, Wojchowski DM and Himmelfarb J: JAK2/Y343/ STAT5 signaling axis is required for erythropoietin-mediated protection against ischemic injury in primary renal tubular epithelial cells. Am J Physiol Renal Physiol 295: F1689-F1695, 2008.

7. Xu X, Cao Z, Cao B, et al: Carbamylated erythropoietin protects the myocardium from acute ischemia/reperfusion injury through a PI3K/AKT-dependent mechanism. Surgery 146: 506-514, 2009.

8. Van der Putten K, Jie KE, Emans ME, et al: Erythropoietin treatment in patients with combined heart and renal failure: objectives and design of the EPOCARES study. J Nephrol 23: 363-368, 2010.

9. Latini R, Brines M and Fiordaliso F: Do non-hemopoietic effects of erythropoietin play a beneficial role in heart failure? Heart Fail Rev 13: 415-423, 2008.

10. Liu G, Sun Y, Li Z, et al: Apoptosis induced by endoplasmic reticulum stress involved in diabetic kidney disease. Biochem Biophys Res Commun 370: 651-656, 2008.

11. Liu XH, Zhang ZY, Sun S, et al: Ischemic postconditioning protects myocardium from ischemia/reperfusion injury through attenuating endoplasmic reticulum stress. Shock 30: 422-427, 2008.

12. Hiroi T, Wei H, Hough C, et al: Protracted lithium treatment protects against the ER stress elicited by thapsigargin in rat PC12 cells: roles of intracellular calcium, GRP78 and Bcl-2. Pharmacogenomics J 5: 102-111, 2005.

13. Janssen K, Horn S, Niemann MT, et al: Inhibition of the ER $\mathrm{Ca} 2+$ pump forces multidrug-resistant cells deficient in Bak and Bax into necrosis. J Cell Sci 122: 4481-4491, 2009.

14. Ali BR: Is cystic fibrosis-related diabetes an apoptotic consequence of ER stress in pancreatic cells? Med Hypotheses 72: 55-57, 2009.

15. Salminen A, Kauppinen A, Suuronen T, et al: ER stress in Alzheimer's disease: a novel neuronal trigger for inflammation and Alzheimer's pathology. J Neuroinflammation 6: 41, 2009.

16. Arduino DM, Esteves AR, Cardoso SM, et al: Endoplasmic reticulum and mitochondria interplay mediates apoptotic cell death: relevance to Parkinson's disease. Neurochem Int 55: 341-348, 2009.

17. Xin W, Li X, Lu X, et al: Involvement of endoplasmic reticulum stress-associated apoptosis in a heart failure model induced by chronic myocardial ischemia. Int J Mol Med 27: 503-509, 2011.

18. Kumar S, Kasseckert S, Kostin S, et al: Ischemic acidosis causes apoptosis in coronary endothelial cells through activation of caspase-12. Cardiovasc Res 73: 172-180, 2007.

19. Yada T, Shimokawa H, Hiramatsu O, et al: Erythropoietin enhances hydrogen peroxide-mediated dilatation of canine coronary collateral arterioles during myocardial ischemia in dogs in vivo. Am J Physiol Heart Circ Physiol 299: H1928-H1935, 2010.

20. Hirata A, Minamino T, Asanuma H, et al: Erythropoietin just before reperfusion reduces both lethal arrhythmias and infarct size via the phosphatidylinositol-3 kinase-dependent pathway in canine hearts. Cardiovasc Drugs Ther 19: 33-40, 2005.

21. Shi Z, Hodges VM, Dunlop EA, et al: Erythropoietin-induced activation of the JAK2/STAT5, PI3K/AKT, AND RAS/ERK pathways promotes malignant cell behavior in a modified breast cancer cell line. Mol Cancer Res 8: 615-626, 2010 